\title{
AUTOPARAMETRIC VIBRATIONS OF A NONLINEAR SYSTEM WITH PENDULUM
}

\author{
J. WARMINSKI AND K. KECIK
}

Received 31 December 2004; Revised 18 May 2005; Accepted 11 July 2005

Vibrations of a nonlinear oscillator with an attached pendulum, excited by movement of its point of suspension, have been analysed in the paper. The derived differential equations of motion show that the system is strongly nonlinear and the motions of both subsystems, the pendulum and the oscillator, are strongly coupled by inertial terms, leading to the so-called autoparametric vibrations. It has been found that the motion of the oscillator, forced by an external harmonic force, has been dynamically eliminated by the pendulum oscillations. Influence of a nonlinear spring on the vibration absorption near the main parametric resonance region has been carried out analytically, whereas the transition from regular to chaotic vibrations has been presented by using numerical methods. A transmission force on the foundation for regular and chaotic vibrations is presented as well.

Copyright (c) 2006 J. Warminski and K. Kecik. This is an open access article distributed under the Creative Commons Attribution License, which permits unrestricted use, distribution, and reproduction in any medium, provided the original work is properly cited.

\section{Introduction}

Vibrations of a pendulum excited by different reasons have been analysed in many papers in different aspects $[1,8]$. Published results show that even the simplest single pendulum structure, forced by an external force or by a moving point of its suspension, can lead to very interesting and unexpected results $[4,9]$. Among the nonlinear systems, we can mention a special class of models which consists of at least two subsystems. If the subsystems are coupled by inertial terms [2], then periodic vibrations generated by one subsystem become an excitation source for the other. This phenomenon performs the socalled autoparametric vibrations [8]. This specific coupling can lead to energy transfer between different vibration modes [6], as well as, to resonances possible only in this specific problem [4]. Moreover, additional types of resonance, internal or combination, are possible [3] and, for some conditions, the system can transit to chaotic motion $[2,6]$. The vibration absorption problem for a single or multiple pendulums systems is presented in

Hindawi Publishing Corporation

Mathematical Problems in Engineering

Volume 2006, Article ID 80705, Pages 1-19

DOI 10.1155/MPE/2006/80705 


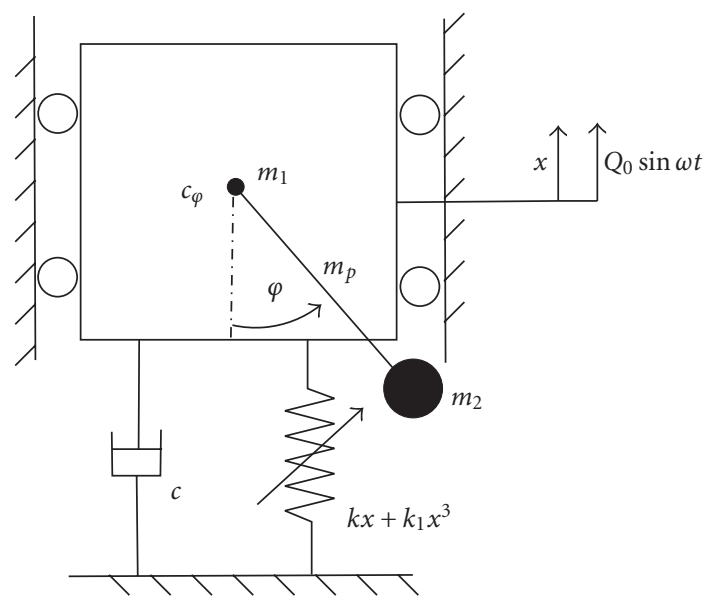

Figure 2.1. Physical model of the system.

$[7,10]$. Additional effects during transition through the resonance regions can appear if a source of excitation has limited power [11], a nonideal problem.

The purpose of this paper is to show possible oscillations for realistic data of the oscillator-pendulum system, taking into account nonlinear stiffness of the supporting spring. The problem of minimisation of the vibrations near the main parametric resonance for regular and chaotic motions and for different spring characteristics is presented.

\section{Model of the vibrating system}

The considered mechanical model, presented in Figure 2.1, is composed of two subsystems: a nonlinear oscillator and a pendulum, made up of two masses $m_{p}$ and $m_{2}$, and attached in a bearing to the mass $m_{1}$. The oscillator is forced by an external harmonic force and is supported by a nonlinear spring having Duffing's type $k x+k_{1} x^{3}$ characteristic, and a linear viscous damper with damping coefficient $c$.

The motion of the system is represented by two generalised coordinates $x$ and $\varphi$, for the oscillator and the pendulum, respectively. Differential equations of motion are derived by applying Lagrange's equations of the second kind. Kinetic energy $T$, potential energy $V$, dissipation function $D$, and the generalised external force $Q$ of the system, presented in Figure 2.1, take the forms

$$
\begin{aligned}
& T=\frac{1}{2} m_{1} \dot{x}^{2}+\frac{1}{2} m_{p}\left(\dot{\varphi}^{2} \frac{l^{2}}{4}+\dot{x}^{2}+2 \dot{x} \dot{\varphi} \frac{l}{2} \sin \varphi\right)+\frac{1}{2} I_{p} \dot{\varphi}^{2}+\frac{1}{2} m_{2}\left(\dot{\varphi}^{2} l^{2}+\dot{x}^{2}+2 \dot{x} \dot{\varphi} l \sin \varphi\right), \\
& V=\frac{1}{2} k x^{2}+\frac{1}{4} k_{1} x^{4}-g l \cos \varphi\left(m_{2}+\frac{1}{2} m_{p}\right), \quad D=\frac{1}{2} c \dot{x}^{2}+\frac{1}{2} c_{\varphi} \dot{\varphi}^{2}, \quad Q=Q_{0} \sin \omega t,
\end{aligned}
$$

where $l$ is the length of the pendulum, $c_{\varphi}$ means a coefficient of viscous damping of the pendulum in its point of suspension, $I_{p}=(1 / 3) m_{p} l^{2}$ is mass moment of inertia of the rod 
having mass $m_{p}, m_{2}$ is a small mass at the tip of the pendulum, and $Q_{0}$ and $\omega$ are the amplitude and frequency of an external harmonic force.

Substituting functions (2.1) into Lagrange's equations of the second kind, we receive differential equations of motion

$$
\begin{gathered}
\left(m_{1}+m_{2}+m_{p}\right) \ddot{x}+c \dot{x}+k x+k_{1} x^{3}+\left(m_{2}+\frac{1}{2} m_{p}\right)\left(\ddot{\varphi} \sin \varphi+\dot{\varphi}^{2} \cos \varphi\right) l=Q_{0} \sin \omega t \\
\left(m_{2}+\frac{1}{3} m_{p}\right) l^{2} \ddot{\varphi}+c_{\varphi} \dot{\varphi}+\left(m_{2}+\frac{1}{2} m_{p}\right)(\ddot{x}+g) l \sin \varphi=0 .
\end{gathered}
$$

Introducing dimensionless time $\tau=\omega_{0} t$, where $\omega_{0}=\sqrt{k /\left(m_{1}+m_{2}+m_{p}\right)}$ is the natural frequency of the oscillator, $X=x / x_{\text {st }}, \varphi \equiv \varphi$ are dimensionless coordinates, and $x_{\text {st }}=$ $\left(m_{1}+m_{2}+m_{3}\right) g / k$ is a static displacement of the linear oscillator, we express (2.2) in dimensionless forms

$$
\begin{gathered}
\ddot{X}+\alpha_{1} \dot{X}+X+\gamma X^{3}+\lambda_{1}\left(\ddot{\varphi} \sin \varphi+\dot{\varphi}^{2} \cos \varphi\right)=q \sin \vartheta \tau, \\
\ddot{\varphi}+\alpha_{2} \dot{\varphi}+\lambda_{2}(\ddot{X}+1) \sin \varphi=0,
\end{gathered}
$$

where dimensionless parameters $\alpha_{1}, \alpha_{2}, \gamma, \lambda_{1}, \lambda_{2}, q, \vartheta$ take definitions

$$
\begin{gathered}
\alpha_{1}=\frac{c}{\left(m_{1}+m_{2}+m_{p}\right) \omega_{0}}, \quad \alpha_{2}=\frac{c_{\varphi}}{\left(m_{2}+(1 / 3) m_{p}\right) l^{2}} \omega_{0}, \quad \gamma=\frac{k_{1}}{k} x_{\mathrm{st}}^{2}, \\
\lambda_{1}=\frac{\left(m_{2}+(1 / 2) m_{p}\right) l}{\left(m_{1}+m_{2}+m_{p}\right) x_{\mathrm{st}}}, \quad \lambda_{2}=\frac{\left(m_{2}+(1 / 2) m_{p}\right) x_{\mathrm{st}}}{\left(m_{2}+(1 / 3) m_{p}\right) l}, \quad q=\frac{Q_{0}}{k x_{\mathrm{st}}}, \quad \vartheta=\frac{\omega}{\omega_{0}} .
\end{gathered}
$$

The dimensionless natural frequency of the linear oscillator is reduced to one.

\section{Analytical solutions}

Differential equations (2.3) and (2.4) are coupled by inertial terms and by nonlinear terms produced by the pendulum motion (terms multiplied by $\lambda_{1}$ and $\lambda_{2}$ ). Moreover, additional nonlinearity is caused by the spring characteristic, parameter $\gamma$. Therefore, to get analytical solutions, it has been assumed that the swing of the pendulum is not large and that the spring is not strongly nonlinear. On the basis of these assumptions, the nonlinear functions $\sin \varphi$ and $\cos \varphi$ are expanded in Taylor series around the lower steady state, for $\varphi \approx 0$. Taking into account the third-order terms we get

$$
\sin \varphi=\varphi-\frac{\varphi^{3}}{6}+0\left(\varphi^{4}\right), \quad \cos \varphi=1-\frac{\varphi^{2}}{2}+0\left(\varphi^{4}\right) .
$$

If the exciting force is harmonic, we can expect that vibrations of the oscillator are harmonic with the same frequency, then the periodic term produced by (2.3) acts as the parametric excitation in (2.4). Therefore, we assume that the oscillator vibrates with frequency $\vartheta$, but the frequency of the pendulum equals $\vartheta / 2$. It means that pendulum vibrates 
under the principal parametric resonance condition. Thus, the solutions are assumed as

$$
\begin{aligned}
& x(\tau)=B_{1}(\tau) \cos \vartheta \tau+B_{2}(\tau) \sin \vartheta \tau, \\
& \varphi(\tau)=C_{1}(\tau) \cos \frac{\vartheta}{2} \tau+C_{2}(\tau) \sin \frac{\vartheta}{2} \tau
\end{aligned}
$$

where $B_{1}(\tau), B_{2}(\tau), C_{1}(\tau), C_{2}(\tau)$ are slowly changing in time functions that represent the oscillator's and pendulum's amplitudes, which are defined as $B=\sqrt{B_{1}^{2}+B_{2}^{2}}, C=\sqrt{C_{1}^{2}+C_{2}^{2}}$, respectively.

Substituting solutions (3.2) into (2.3), (2.4), taking into account (3.1), and next balancing the harmonic terms $\cos \vartheta \tau, \sin \vartheta \tau, \cos (\vartheta / 2) \tau, \sin (\vartheta / 2) \tau$, we get a set of the firstorder approximate differential equations:

$$
\begin{aligned}
&-2 \vartheta \dot{B}_{1}+\alpha_{1} \dot{B}_{2}+\lambda_{1} \vartheta \dot{C}_{1} C_{1}\left(\frac{C_{1}^{2}}{6}-1\right)+\lambda_{1} \vartheta \dot{C}_{2} C_{2}\left(1-\frac{C_{2}^{2}}{6}\right)-\alpha_{1} \vartheta B_{1}+B_{2}\left(1-\vartheta^{2}\right) \\
&+\frac{1}{2} \lambda_{1} \vartheta^{2} C_{1} C_{2}\left(\frac{C_{2}^{2}}{12}-1\right)+\frac{1}{24} \lambda_{1} \vartheta^{2} C_{1}^{3} C_{2}+\frac{3}{4} \gamma B_{2}\left(B_{1}^{2}+B_{2}^{2}\right)-q=0, \\
& \alpha_{1} \dot{B}_{1}+ 2 \vartheta \dot{B}_{2}+\lambda_{1} \vartheta \dot{C}_{1} C_{2}\left(1-\frac{C_{1}^{2}}{4}-\frac{C_{2}^{2}}{12}\right)+\lambda_{1} \vartheta \dot{C}_{2} C_{1}\left(1-\frac{C_{1}^{2}}{12}-\frac{C_{2}^{2}}{4}\right)+\alpha_{1} \vartheta B_{2}+B_{1}\left(1-\vartheta^{2}\right) \\
&-\frac{1}{4} \lambda_{1} \vartheta^{2}\left(C_{1}^{2}-C_{2}^{2}\right)+\frac{1}{48} \lambda_{1} \vartheta^{2}\left(C_{1}^{4}-C_{2}^{4}\right)+\frac{3}{4} \gamma B_{1}\left(B_{1}^{2}+B_{2}^{2}\right)=0, \\
& \lambda_{2} \vartheta \dot{B}_{1} C_{1}\left(\frac{C_{1}^{2}}{12}+\frac{C_{2}^{2}}{4}-1\right)+\lambda_{2} \vartheta \dot{B}_{2} C_{2}\left(\frac{C_{2}^{2}}{6}-1\right)-\vartheta \dot{C}_{1}+\alpha_{2} \dot{C}_{2}+\frac{1}{2} \lambda_{2} \vartheta^{2} B_{1} C_{2}\left(1-\frac{C_{2}^{2}}{6}\right) \\
&+\frac{1}{2} \lambda_{2} \vartheta^{2} B_{2} C_{1}\left(\frac{C_{1}^{2}}{12}+\frac{C_{2}^{2}}{4}-1\right)-\frac{1}{2} \alpha_{2} \vartheta C_{1}+\left(\lambda_{2}-\frac{\vartheta^{2}}{4}\right) C_{2}-\frac{1}{8} \lambda_{2} C_{2}\left(C_{1}^{2}+C_{2}^{2}\right)=0, \\
& \lambda_{2} \vartheta \dot{B}_{1} C_{2}\left(\frac{C_{1}^{2}}{4}+\frac{C_{2}^{2}}{12}-1\right)+\lambda_{2} \vartheta \dot{B}_{2} C_{1}\left(1-\frac{C_{1}^{2}}{6}\right)+\alpha_{2} \dot{C}_{1}+\vartheta \dot{C}_{2}+\frac{1}{2} \lambda_{2} \vartheta^{2} B_{1} C_{1}\left(\frac{C_{1}^{2}}{6}-1\right) \\
&+\frac{1}{2} \lambda_{2} \vartheta^{2} B_{2} C_{2}\left(\frac{C_{1}^{2}}{4}+\frac{C_{2}^{2}}{12}-1\right)+\frac{1}{2} \alpha_{2} \vartheta C_{2}+\left(\lambda_{2}-\frac{\vartheta^{2}}{4}\right) C_{1}-\frac{1}{8} \lambda_{2} C_{1}\left(C_{1}^{2}+C_{2}^{2}\right)=0 .
\end{aligned}
$$

The derivatives of the second order and terms having derivatives in a power higher than the one in (3.3) are neglected.

For a steady state, the first-order derivative of the amplitudes is equal to zero

$$
\dot{B}_{1}=0, \quad \dot{B}_{2}=0, \quad \dot{C}_{1}=0, \quad \dot{C}_{2}=0 .
$$

Moreover, according to [7], for small oscillations of the pendulum, we can assume that $C^{2} / 12 \approx 0, C^{2} / 6 \approx 0$. Then we receive four algebraic equations with unknown amplitudes 
$B_{1}, B_{2}, C_{1}, C_{2}$,

$$
\begin{gathered}
-\alpha_{1} \vartheta B_{1}+B_{2}\left(1-\vartheta^{2}\right)+\frac{3}{4} \gamma B_{2}\left(B_{1}^{2}+B_{2}^{2}\right)-\frac{1}{2} \lambda_{1} \vartheta^{2} C_{1} C_{2}-q=0, \\
\alpha_{1} \vartheta B_{2}+B_{1}\left(1-\vartheta^{2}\right)+\frac{3}{4} \gamma B_{1}\left(B_{1}^{2}+B_{2}^{2}\right)-\frac{1}{4} \lambda_{1} \vartheta^{2}\left(C_{1}^{2}-C_{2}^{2}\right)=0, \\
-\frac{1}{2} \alpha_{2} \vartheta C_{1}+C_{2}\left(\lambda_{2}-\frac{1}{4} \vartheta^{2}\right)-\frac{1}{2} \lambda_{2} \vartheta^{2}\left(B_{2} C_{1}-B_{1} C_{2}\right)-\frac{1}{8} \lambda_{2} C_{2}\left(C_{1}^{2}+C_{2}^{2}-B_{2} C_{1} C_{2} \vartheta^{2}\right)=0, \\
\frac{1}{2} \alpha_{2} \vartheta C_{2}+C_{1}\left(\lambda_{2}-\frac{1}{4} \vartheta^{2}\right)-\frac{1}{2} \lambda_{2} \vartheta^{2}\left(B_{1} C_{1}+B_{2} C_{2}\right)-\frac{1}{8} \lambda_{2} C_{1}\left(C_{1}^{2}+C_{2}^{2}-B_{2} C_{1} C_{2} \vartheta^{2}\right)=0 .
\end{gathered}
$$

The set of the above algebraic equations may have the following solutions:

(a) trivial $B_{1}=B_{2}=C_{1}=C_{2}=0$, only if $q=0$;

(b) semitrivial $B_{1} \neq 0, B_{2} \neq 0, C_{1}=C_{2}=0$, oscillator vibrates, pendulum does not swing;

(c) semitrivial $B_{1}=0, B_{2}=0, C_{1} \neq 0, C_{2} \neq 0$, oscillator does not vibrate, pendulum swings;

(d) nontrivial $B_{1} \neq 0, B_{2} \neq 0, C_{1} \neq 0, C_{2} \neq 0$, both oscillator and pendulum vibrate.

The semitrivial and nontrivial solutions have been solved numerically from the set of nonlinear algebraic equations (3.5).

If we assume that damping of the pendulum is equal to zero $\alpha_{2}=0$, we can find a condition of the full elimination of the oscillator vibrations. Putting $B_{1}=0, B_{2}=0$, and $\alpha_{2}=0$ in (3.5), in the first-order approximation, we get

$$
\vartheta^{*}=\sqrt{2 \lambda_{2}+\lambda_{2} \sqrt{4-\frac{2 q}{\lambda_{1} \lambda_{2}}}}, \quad C^{*}=\sqrt{2} \sqrt{2-\sqrt{4-\frac{2 q}{\lambda_{1} \lambda_{2}}}},
$$

where $9^{*}$ is the frequency of the full vibration absorption of the oscillator and $C^{*}$ is the amplitude of the pendulum. This state can be achieved only if the damping of the pendulum equals zero. We can notice that nonlinear characteristic of the spring does not influence this condition.

If the pendulum does not swing, which corresponds to semitrivial solutions, case (b), we can determine a resonance curve of the nonlinear one degree of freedom system with joined masses $m=m_{1}+m_{2}+m_{p}$. Putting $C_{1}=0$ and $C_{2}=0$ in (3.5), after rearrangements, we get the resonance curve

$$
\frac{9}{16} \gamma^{2} B^{6}+\frac{3}{2} \gamma\left(1-\vartheta^{2}\right) B^{4}+\left[\left(1-\vartheta^{2}\right)^{2}+\alpha_{1}^{2} \vartheta^{2}\right] B^{2}-q^{2}=0
$$

If $\gamma=0$, then

$$
B=\frac{q}{\sqrt{\left(1-\vartheta^{2}\right)^{2}+\alpha_{1}^{2} \vartheta^{2}}}
$$

is the amplitude of the linear oscillator. 


\section{Solutions stability}

Analysis of stability of the semitrivial and nontrivial solutions is carried out by using the approximate equations (3.3). Determining derivatives $\dot{B}_{1}, \dot{B}_{2}, \dot{C}_{1}, \dot{C}_{2}$ the so-called amplitude modulation equations have been found:

$$
\begin{array}{ll}
\dot{B}_{1}=f_{1}\left(B_{1}, B_{2}, C_{1}, C_{2}\right), & \dot{B}_{2}=f_{2}\left(B_{1}, B_{2}, C_{1}, C_{2}\right), \\
\dot{C}_{1}=f_{3}\left(B_{1}, B_{2}, C_{1}, C_{2}\right), & \dot{C}_{2}=f_{4}\left(B_{1}, B_{2}, C_{1}, C_{2}\right) .
\end{array}
$$

Functions $f_{1}\left(B_{1}, B_{2}, C_{1}, C_{2}\right), f_{2}\left(B_{1}, B_{2}, C_{1}, C_{2}\right), f_{3}\left(B_{1}, B_{2}, C_{1}, C_{2}\right), f_{4}\left(B_{1}, B_{2}, C_{1}, C_{2}\right)$ are expressed as

$$
f_{1}=\frac{W_{\dot{B}_{1}}}{W}, \quad f_{2}=\frac{W_{\dot{B}_{2}}}{W}, \quad f_{3}=\frac{W_{\dot{C}_{1}}}{W}, \quad f_{4}=\frac{W_{\dot{C}_{2}}}{W},
$$

where

$$
\begin{gathered}
W=\left|\begin{array}{llll}
a_{11} & a_{12} & a_{13} & a_{14} \\
a_{21} & a_{22} & a_{23} & a_{24} \\
a_{31} & a_{32} & a_{33} & a_{34} \\
a_{41} & a_{42} & a_{43} & a_{44}
\end{array}\right|, \quad W_{\dot{B}_{1}}=\left|\begin{array}{llll}
a_{0} & a_{12} & a_{13} & a_{14} \\
b_{0} & a_{22} & a_{23} & a_{24} \\
c_{0} & a_{32} & a_{33} & a_{34} \\
d_{0} & a_{42} & a_{43} & a_{44}
\end{array}\right|, \\
W_{\dot{B}_{2}}=\left|\begin{array}{llll}
a_{11} & a_{0} & a_{13} & a_{14} \\
a_{21} & b_{0} & a_{23} & a_{24} \\
a_{31} & c_{0} & a_{33} & a_{34} \\
a_{41} & d_{0} & a_{43} & a_{44}
\end{array}\right|, \quad W_{\dot{C}_{1}}=\left|\begin{array}{llll}
a_{11} & a_{12} & a_{0} & a_{14} \\
a_{21} & a_{22} & b_{0} & a_{24} \\
a_{31} & a_{32} & c_{0} & a_{34} \\
a_{41} & a_{42} & d_{0} & a_{44}
\end{array}\right|, \\
W_{\dot{C}_{2}}=\left|\begin{array}{llll}
a_{11} & a_{12} & a_{13} & a_{0} \\
a_{21} & a_{22} & a_{23} & b_{0} \\
a_{31} & a_{32} & a_{33} & c_{0} \\
a_{41} & a_{42} & a_{43} & d_{0}
\end{array}\right| .
\end{gathered}
$$

Coefficients applied in the above determinants are defined as

$$
\begin{gathered}
a_{11}=-2 \vartheta, \quad a_{12}=\alpha_{1}, \quad a_{13}=-\lambda_{1} \vartheta C_{1}, \quad a_{14}=\lambda_{1} \vartheta C_{2}, \\
a_{21}=\alpha_{1}, \quad a_{22}=2 \vartheta, \quad a_{23}=\lambda_{1} \vartheta C_{2}\left(1-\frac{C_{1}^{2}}{4}\right), \quad a_{24}=\lambda_{1} \vartheta C_{1}\left(1-\frac{C_{2}^{2}}{4}\right), \\
a_{31}=-\lambda_{2} \vartheta C_{1}\left(1-\frac{C_{2}^{2}}{4}\right), \quad a_{32}=-\lambda_{2} \vartheta C_{2}, \quad a_{33}=-\vartheta, \quad a_{34}=\alpha_{2}, \\
a_{41}=-\lambda_{2} \vartheta C_{2}\left(1-\frac{C_{1}^{2}}{4}\right), \quad a_{42}=\lambda_{2} \vartheta C_{1}, \quad a_{43}=\alpha_{2}, \quad a_{44}=\vartheta, \\
a_{0}=\alpha_{1} \vartheta B_{1}-B_{2}\left(1-\vartheta^{2}\right)-\frac{3}{4} \gamma B_{2}\left(B_{1}^{2}+B_{2}^{2}\right)+\frac{1}{2} \lambda_{1} \vartheta{ }^{2} C_{1} C_{2}+q,
\end{gathered}
$$




$$
\begin{gathered}
b_{0}=-\alpha_{1} \vartheta B_{2}-B_{1}\left(1-\vartheta^{2}\right)-\frac{3}{4} \gamma B_{1}\left(B_{1}^{2}+B_{2}^{2}\right)+\frac{1}{4} \lambda_{1} \vartheta^{2}\left(C_{1}^{2}-C_{2}^{2}\right), \\
c_{0}=\frac{1}{2} \alpha_{2} \vartheta C_{2}-C_{1}\left(\lambda_{2}-\frac{1}{4} \vartheta^{2}\right)-\frac{1}{2} \lambda_{2} \vartheta^{2}\left(B_{1} C_{1}+B_{2} C_{2}\right)-\frac{1}{8} \lambda_{2} C_{1}\left(C_{1}^{2}+C_{2}^{2}-B_{2} C_{1} C_{2} \vartheta^{2}\right), \\
d_{0}=-\frac{1}{2} \alpha_{2} \vartheta C_{2}-C_{1}\left(\lambda_{2}-\frac{1}{4} \vartheta^{2}\right)+\frac{1}{2} \lambda_{2} \vartheta^{2}\left(B_{2} C_{1}-B_{1} C_{2}\right)+\frac{1}{8} \lambda_{2} C_{1}\left(C_{1}^{2}+C_{2}^{2}-B_{2} C_{1} C_{2} \vartheta^{2}\right) .
\end{gathered}
$$

Perturbing the analysed solutions, $B_{1}+\delta B_{1}, B_{2}+\delta B_{2}, C_{1}+\delta C_{1}, C_{2}+\delta C_{2}$, next substituting them to (4.1), then subtracting from unperturbed equations, and taking into account linear part of their expansions in power series, we get a set of linear differential equations in variations $\delta \dot{B}_{1}, \delta \dot{B}_{2}, \delta \dot{C}_{1}, \delta \dot{C}_{2}$. Stability of the system solutions depends on the eigenvalues of the Jacobian

$$
[J]=\left[\begin{array}{llll}
\frac{\partial f_{1}}{\partial B_{1}} & \frac{\partial f_{1}}{\partial B_{2}} & \frac{\partial f_{1}}{\partial C_{1}} & \frac{\partial f_{1}}{\partial C_{2}} \\
\frac{\partial f_{2}}{\partial B_{1}} & \frac{\partial f_{2}}{\partial B_{2}} & \frac{\partial f_{2}}{\partial C_{1}} & \frac{\partial f_{2}}{\partial C_{2}} \\
\frac{\partial f_{3}}{\partial B_{1}} & \frac{\partial f_{3}}{\partial B_{2}} & \frac{\partial f_{3}}{\partial C_{1}} & \frac{\partial f_{3}}{\partial C_{2}} \\
\frac{\partial f_{4}}{\partial B_{1}} & \frac{\partial f_{4}}{\partial B_{2}} & \frac{\partial f_{4}}{\partial C_{1}} & \frac{\partial f_{4}}{\partial C_{2}}
\end{array}\right]
$$

whose elements denote the partial derivatives calculated adequately for semi- or nontrivial solutions.

\section{Exemplary results}

Numerical analysis of the system has been carried out on the basis of data taken from the realistic system, for which the dimensionless parameters take values:

$$
\alpha_{1}=0.49, \quad \alpha_{2}=0.015, \quad \lambda_{1}=1.39, \quad \lambda_{2}=0.17, \quad q=0.1
$$

The vibration amplitudes of the oscillator and the pendulum have been found by solving (3.5).

Analytical results have been verified by a direct numerical integration of (2.3) and (2.4). Numerical procedures, based on fourth-order Runge-Kutta method, have been prepared in Fortran language and in Matlab-Simulink package, taking into account the error control. The bifurcation diagrams, basins of attraction, and the Laypunov exponents (presented in Sections 5 and 6) have been obtained by methods and procedures published in [5]. Numerical simulations have been done on Unix Digital Alpha Station by using packages submitted in [5] by Hunt and Kostelich.

Amplitudes versus excitation frequency $\vartheta$ of the oscillator and the pendulum, obtained for the linear spring $\gamma=0$ and for the pendulum without damping $\alpha_{2}=0$, are presented in Figures 5.1(a) and 5.1(b), respectively. Solid lines denote stable solutions while dashed 


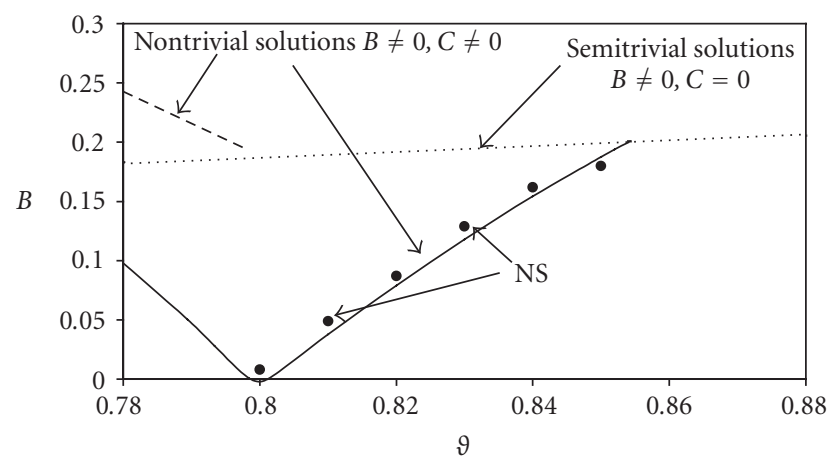

(a)

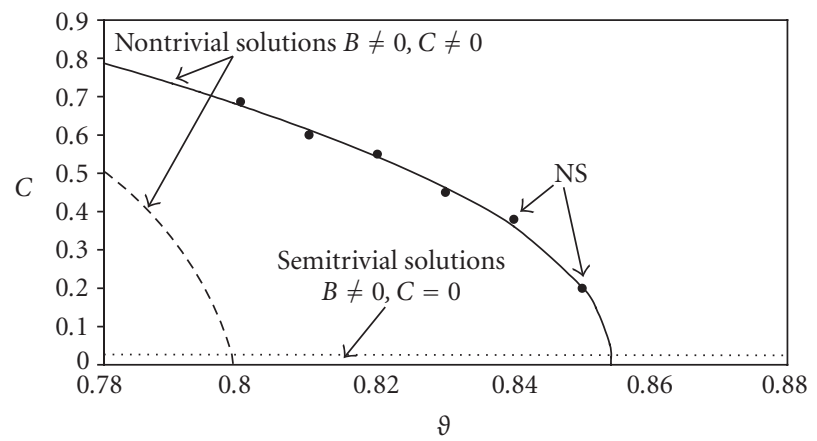

(b)

Figure 5.1. Amplitude versus excitation frequency for $\gamma=0, \alpha_{2}=0$; (a) oscillator, (b) pendulum.

ones unstable, and black points present direct numerical simulation results. In Figure 5.2, for comparison, the bifurcation diagram has been plotted for the same parameters.

The sampling frequency in this diagram is equal to the excitation frequency and initial conditions have been fixed $X=0, \dot{X}=0, \varphi=0.1, \dot{\varphi}=0$. It means that for each point, in the bifurcation diagram, the pendulum has been disturbed from the lower steady state. Comparing Figures 5.1 and 5.2 we can find that analytical results are correct in limited frequency range $\vartheta \in(0.792,0.852)$. The upper branch of the resonance curve of the pendulum obtained analytically is stable in a very wide area (Figure 5.1(b)). However, numerical simulation (NS) results, marked by dots in Figure 5.1, and numerical results presented in Figure 5.2 are confirmed only in a narrow resonance region where nontrivial solutions, corresponding to both oscillator and the pendulum vibrations, may exist. This solution is presented in bifurcation diagram by single solid lines for the oscillator (Figure 5.2(a)) and by two lines for the pendulum (Figure 5.2(b)).

The diagrams confirm the assumption that the oscillator vibrates harmonically with single frequency $\vartheta$, whilst the pendulum oscillates subharmonically with $\vartheta / 2$ frequency. In this resonance area, according to (3.6), the absorption effect takes place about frequency 


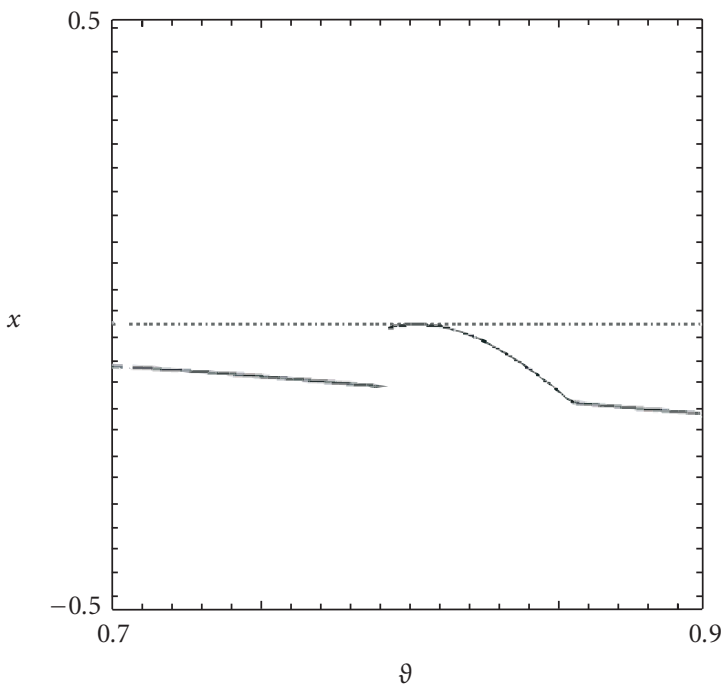

(a)

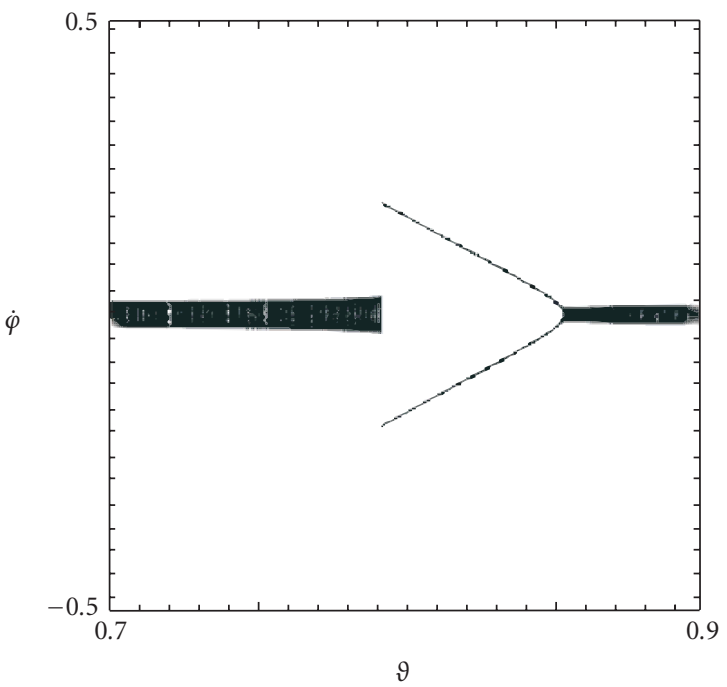

(b)

Figure 5.2. Bifurcation diagrams of the oscillator and the pendulum versus excitation frequency for $\gamma=0, \alpha_{2}=0$; (a) displacement, (b) angular velocity.

$\vartheta^{*}=0.8$, for which vibrations of the oscillator are close to zero. Then, the pendulum vibrates with amplitude $C^{*}=0.67$. Outside the resonance region, the system vibrates quasiperiodically (black areas on bifurcation diagrams). This situation takes place due to lack of pendulum damping, which leads to two, out of four, roots of the Jacobian (4.5), having only imaginary parts. Therefore the pendulum oscillates permanently about its 


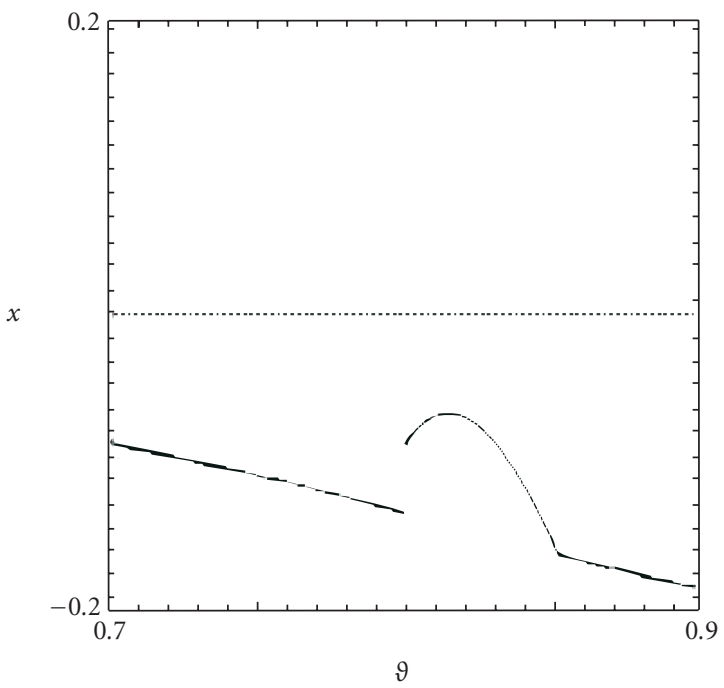

(a)

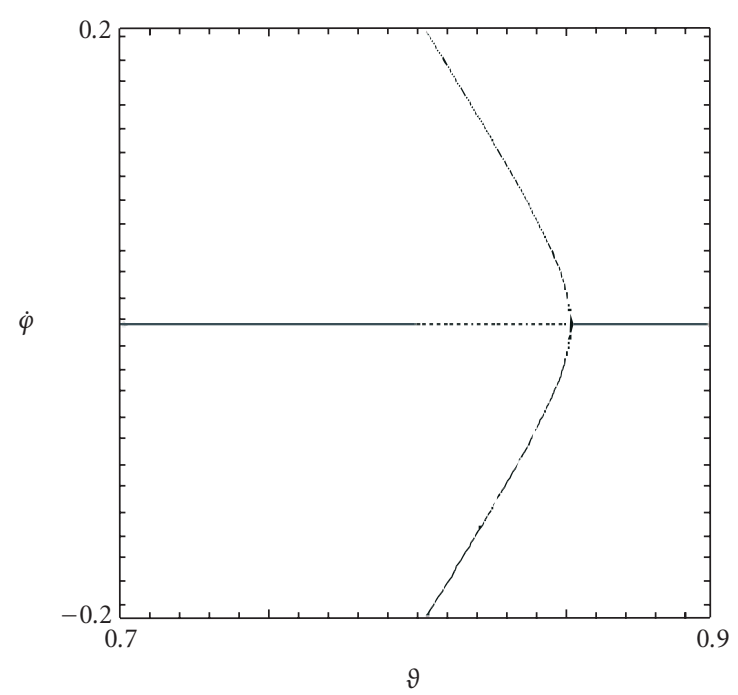

(b)

Figure 5.3. Bifurcation diagrams of displacement of the (a) oscillator and (b) angular velocity of the pendulum versus excitation frequency, for $q=0.1, \alpha_{2}=0.01$, and initial conditions $X=0, \dot{X}=0$, $\varphi=0.1, \dot{\varphi}=0$.

lower position. However, in practical applications, the damping of the pendulum exists and therefore parameter $\alpha_{2}$ has to be put greater than zero.

In Figure 5.3 bifurcation diagrams are plotted for realistic data for which either the pendulum damping or nonlinearity of the spring is assumed as $\alpha_{2}=0.01, \gamma=0.00046$. 


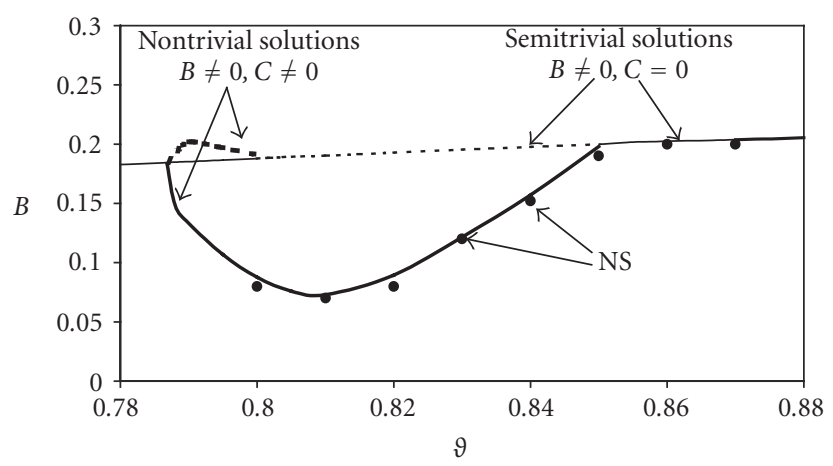

(a)

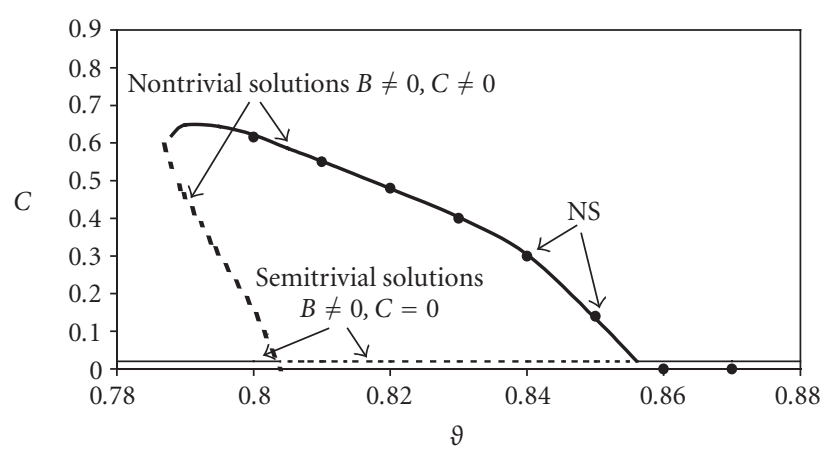

(b)

Figure 5.4. Amplitude versus excitation frequency for $\gamma=0.00046, \alpha_{2}=0.01$; (a) oscillator, (b) pendulum.

Due to existing damping, the quasiperiodic motion disappears. Moreover, solutions obtained analytically correspond to the numerical simulation. The analytical results for these parameters are presented in Figure 5.4. Out of resonance, all roots of the Jacobian (4.5) have negative real parts, it means that the semitrivial solutions $B \neq 0, C=0$ are stable and oscillations of the pendulum disappear. The set, oscillator-pendulum, vibrates as a one-degree-of-freedom system and the absorption effect does not take place, the resonance curve is described by (3.7).

At the ends of the resonance area, nontrivial as well as semitrivial solutions are possible, depending on initial conditions. Basins of attraction of the pendulum in this region, for $\vartheta=0.8$, are presented in Figure 5.5. Attractors no. 1 represent nontrivial solutions and their basins are marked by a light grey colour.

Darker narrow areas correspond to semitrivial solution (attractor no. 2). In these areas where two stable solutions are possible, the nontrivial solutions have much wider basins of attractions. Probability that pendulum will swing is much higher. 


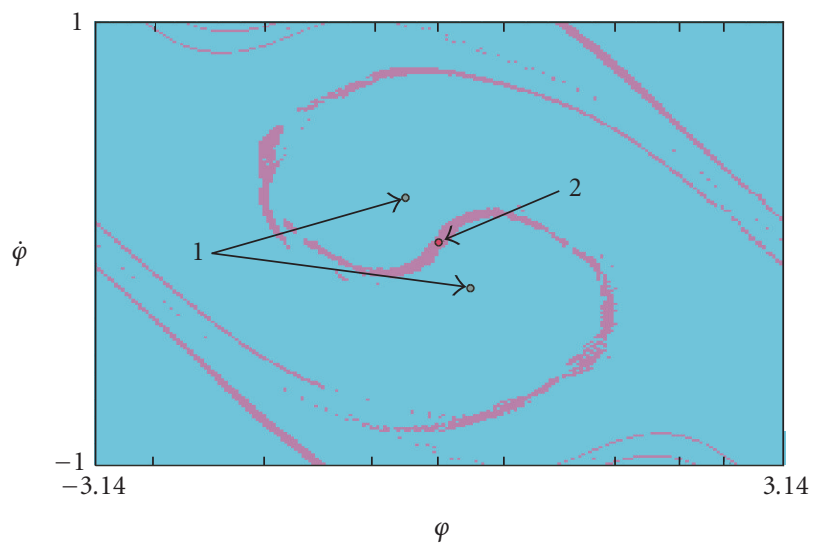

Figure 5.5. Basins of attractions of the pendulum for $\vartheta=0.8$.

The graphs in Figure 5.4 present the influence of a very small positive value of nonlinear term of the spring $(\gamma=0.00046)$ on the vibration amplitude. In spite of the fact that a stiff spring should have a slope oriented in the right-hand side (hard characteristic), the slope of the resonance curve is still oriented in the left-hand side, like for the system with features of the soft characteristic. It means that "soft" nonlinear terms performed by the pendulum dominate. Similar situation has been observed for negative stiffness of the spring $\gamma=-0.0046$ ("soft" spring). This value does not change motion of the system radically. Additional softening due to "soft" spring existence has been negligible.

To present the influence of the "hard" and "soft" characteristics of the spring on the main parametric resonance area, the resonance curves have been calculated for the new set of parameters

$$
\alpha_{1}=0.49, \quad \alpha_{2}=0.015, \quad \lambda_{1}=1.8, \quad \lambda_{2}=0.3, \quad q=0.5
$$

and $\gamma=0$ for linear, $\gamma=0.5$ for stiff, and $\gamma=-0.5$ for soft springs.

Comparing vibration amplitudes of the oscillator having "soft" or "hard" springs with linear one (Figure 5.6(a)), we can notice that minimum of the amplitude (absorption effect) occurs almost at the same place. However, for decreasing frequency below $\vartheta=0.9$, the difference increases. Amplitudes of the soft spring oscillator are higher than the linear or stiff spring system. According to the pendulum motion (Figure 5.6(b)), the soft spring reduces the resonance region, which is not wanted from the absorption point of view, for elimination of the oscillator vibrations the motion of the pendulum is required.

\section{Transition to chaos}

The analytical approach, presented in Section 3, can be applied only for limited frequency range, around the main parametric resonance. The semi- and nontrivial solutions obtained numerically confirm the absorption effect, which takes place around frequency 


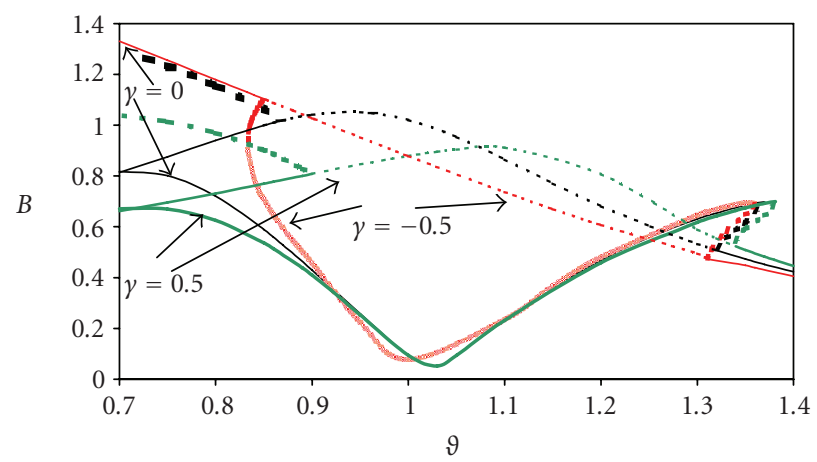

(a)

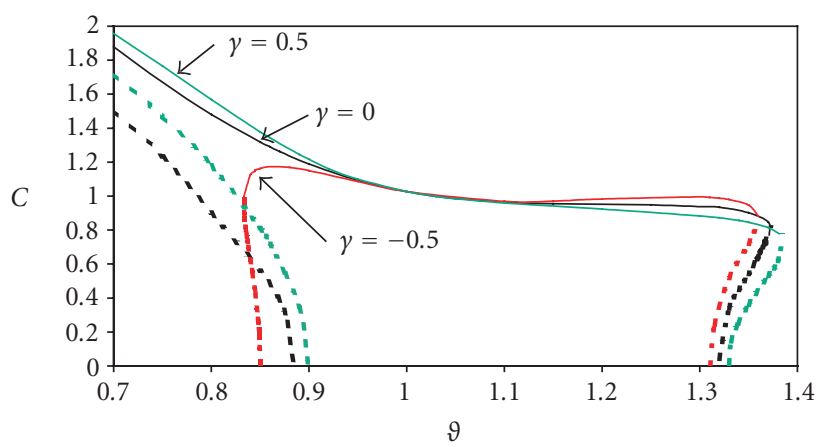

(b)

Figure 5.6. Amplitude versus excitation frequency for various nonlinear springs, $\gamma=0, \gamma=0.5, \gamma=$ $-0.5, \alpha_{2}=0.015$; (a) oscillator, (b) pendulum.

$\vartheta=0.8$ and for small oscillations of the pendulum, which is in accordance with analytical results.

From practical point of view, however, dynamics of the system should be checked when the excitation frequency changes out of the main parametric resonance region. Therefore the influence of two parameters, the amplitude $q$ and the frequency $\vartheta$ of the excitation, has been tested in ranges $q \in\langle 0.1,10\rangle, \vartheta \in\langle 0,10\rangle$.

The behaviour of the model for small excitation amplitude $\left(q=0.1, \alpha_{2}=0\right)$ is presented on bifurcation diagrams in Figure 6.1. Observing the oscillator motion, we can notice local decrease of the amplitude near $\vartheta=0.8$, and next the increase close to the frequency $\vartheta \approx 1$. The oscillations of the pendulum are rather small and only in the neighbourhood of the resonance area a very narrow harmonic motion is possible (Figure 6.1(b)). The behaviour is similar for different initial conditions of the system.

The increase of the external excitation amplitude changes this situation radically. Keeping the same initial conditions $X=0, \dot{X}=0, \varphi=0.1, \dot{\varphi}=0$, and constant excitation frequency $\vartheta=1$, we investigate the influence of the amplitude $q$ (Figure 6.2). Near value of 
14 Autoparametric vibrations

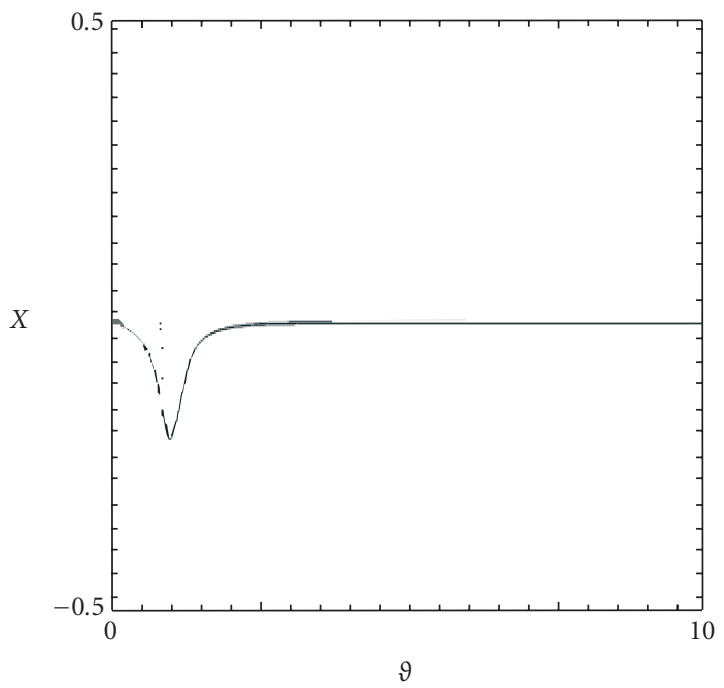

(a)

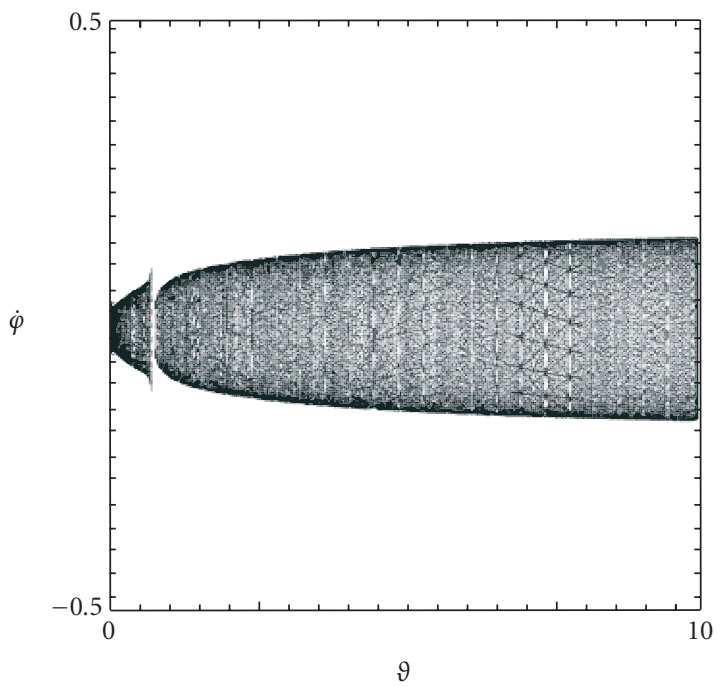

(b)

Figure 6.1. Bifurcation diagrams of displacement of the (a) oscillator and (b) angular velocity of the pendulum versus excitation frequency, for $q=0.1, \alpha_{2}=0$, and initial conditions $X=0, \dot{X}=0, \varphi=0.1$, $\dot{\varphi}=0$.

$q$ over 1.25 , after the period doubling bifurcation, the system transits to chaotic motion if $q>1.4$, it goes back to periodicity and only if $q$ is over 5 , it transits to a very wide region of chaotic vibrations. 


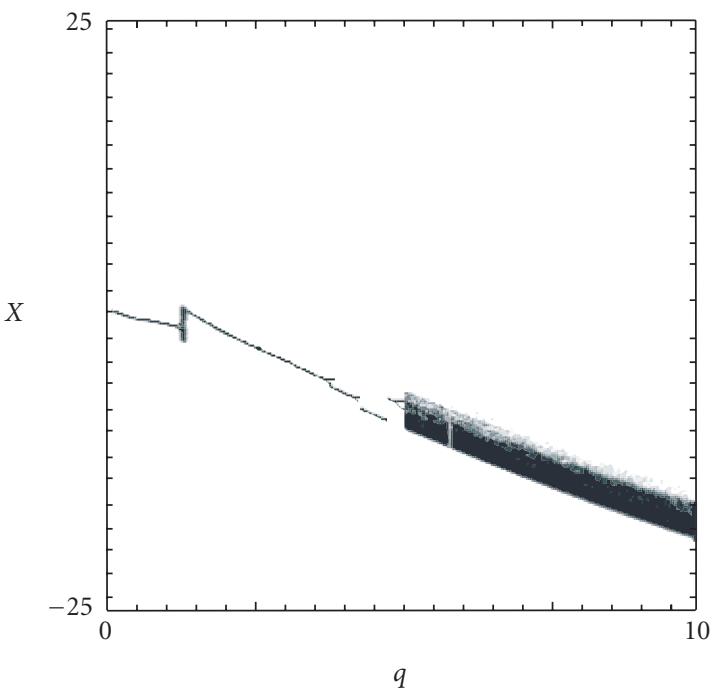

(a)

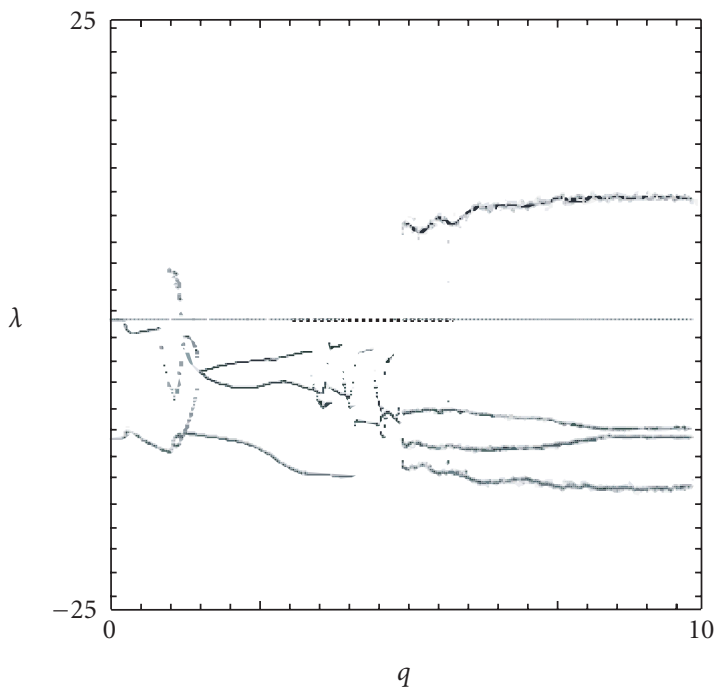

(b)

Figure 6.2. Bifurcation diagrams of displacement of the (a) oscillator and (b) the Lyapunov exponents diagram versus $q$ parameter, $\vartheta=1$ and initial conditions $X=0, \dot{X}=0, \varphi=0.1, \dot{\varphi}=0$.

The nature of motion is confirmed in Figure 6.2(b) where the Lyapunov exponent diagram emphasises both chaotic and regular oscillations (two intervals with positive 


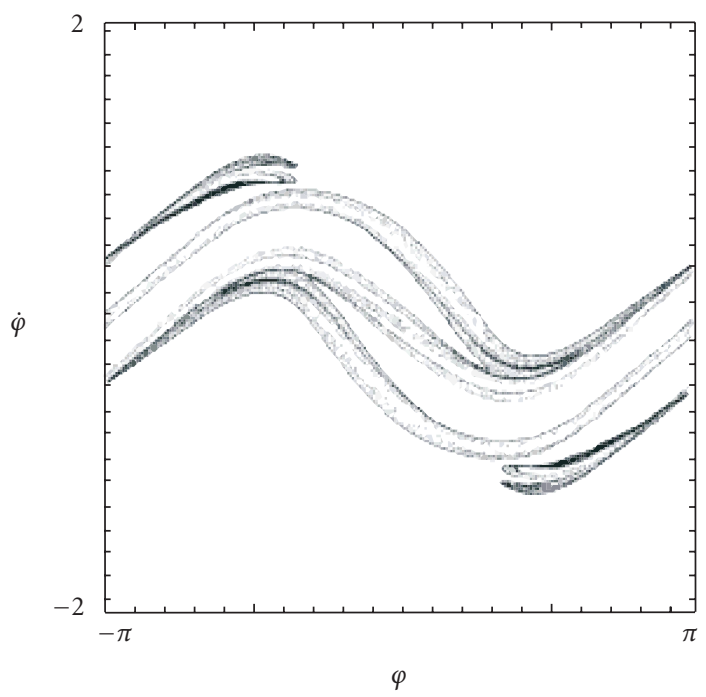

(a)

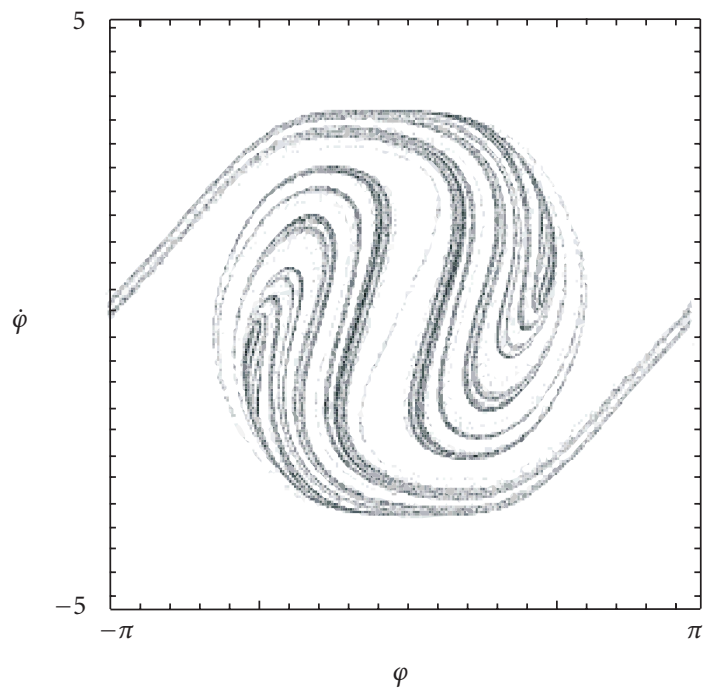

(b)

Figure 6.3. Chaotic attractors on Poincaré maps, for $\vartheta=1, \gamma=4.6 \times 10^{-4}$, and (a) $q=1.3$, (b) $q=10$.

value of the maximal Lyapunov exponent). Chaotic attractors for the first and the second chaotic regions are presented on Poincaré sections in Figures 6.3(a) and 6.3(b), respectively. In both cases chaotic motion is composed of rotation an oscillations of the pendulum. 


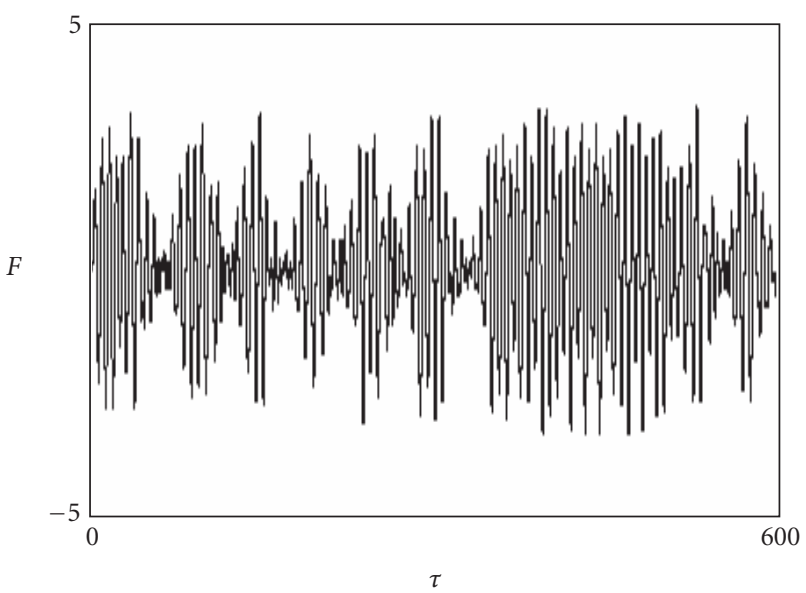

(a)

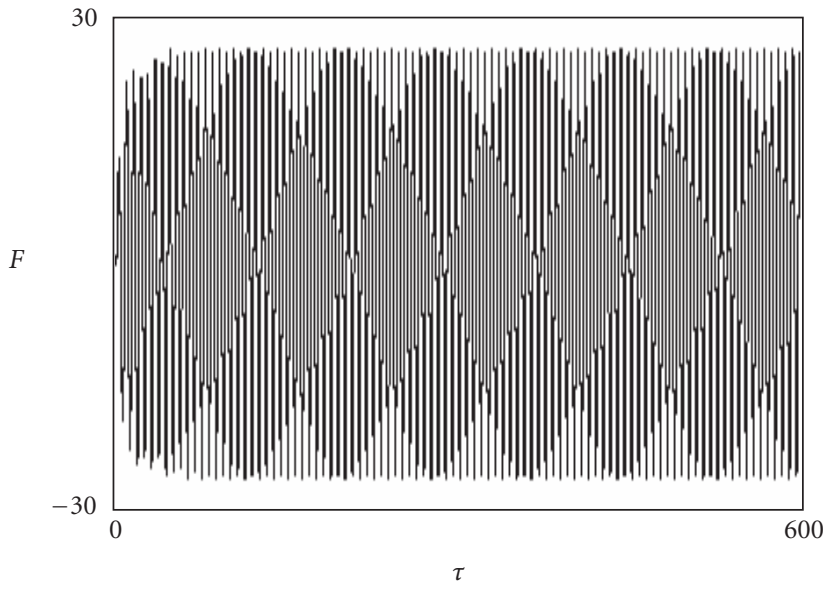

(b)

Figure 6.4. Time histories of the force transmitted on the ground, $\vartheta=1, \gamma=4.6 \times 10^{-4}$, initial conditions: $X=0, \dot{X}=0, \varphi=0.1, \dot{\varphi}=0$, and (a) $q=1.3$, (b) $q=10$.

The chaotic dynamics result in irregular force transmitted on the foundation defined as $F(\tau)=\alpha_{1} \dot{x}+x+\gamma x^{3}$. Time history of the force in Figure 6.4(a) is obtained for excitation of the system $q=1.3$ laying in the first chaotic region, while in Figure 6.4(b) the external excitation is relatively high $q=10$ and lays in the second chaotic region. In such a case the nonlinear stiff spring reduces the vibration amplitudes and the force transmitted on the foundation. 


\section{Conclusions}

Influence of the nonlinear spring on the autoparametric system in the neighbourhood of the main parametric resonance has been investigated. It has been shown that the vibration absorption effect may exist inside the resonance area, and that the differences between linear, soft, and stiff supporting springs are not significant. The results have been found by using analytical the harmonic balance method and next verified numerically. The approximate analytically solutions are in good agreement only if oscillations of the pendulum are damped and are small. From practical point of view, the stiff characteristic of the spring reduces vibration amplitudes if the vibration frequency is detuned from the absorption point. This type of spring is more beneficial than linear or soft springs.

The numerical results presented in this paper show that it is possible to get regular and chaotic motions for the set of realistic data. Chaotic motion can be got by the increase of the amplitude of external excitation. Transition to chaos is preceded by the period doubling bifurcation, and the escape from chaos has rapid nature, preceded by the crisis bifurcation. The first narrow chaotic region appears for relatively small amplitude $q$, while the second one occurs for big value of parameter $q$. The second area is very wide. It leads to transmission of irregular motion on foundation which can change the vibration absorption criterion. Nonlinear stiff spring reduces vibration amplitudes and in consequence the force acting on the ground. In the next step, the results will be verified experimentally and an appropriate criterion to minimise the force transmitted on the environment under regular and chaotic motion conditions will be determined.

\section{References}

[1] D. A. Acheson, A pendulum theorem, Proceedings of the Royal Society of London. Series A 443 (1993), no. 1917, 239-245.

[2] A. K. Bajaj, S. I. Chang, and J. M. Johnson, Amplitude modulated dynamics of a resonantly excited autoparametric two degree-of-freedom system, Nonlinear Dynamics 5 (1994), 433-457.

[3] M. P. Cartmell and J. W. Roberts, Simultaneous combination resonances in an autoparametrically resonant system, Journal of Sound and Vibration 123 (1988), no. 1, 81-101.

[4] W. K. Lee and C. S. Hsu, A global analysis of an harmonically excited spring-pendulum system with internal resonance, Journal of Sound and Vibration 171 (1994), no. 3, 335-359.

[5] H. E. Nusse and J. A. Yorke, Dynamics: Numerical Explorations, Applied Mathematical Sciences, vol. 101, Springer, New York, 1994.

[6] D. Sado, Energy Transfer in Nonlinearly Coupled Systems with Two Degrees of Freedom (Przenoszenie energii w nieliniowo sprzężonych układach o dwóch stopniach swobody), Prace Naukowe, Mechanika, z.166, Oficyna Wydawnicza Politechniki Warszawskiej, Warszawa, 1997 (in Polish).

[7] Y. Song, H. Sato, Y. Iwata, and T. Komatsuzaki, The response of a dynamic vibration absorber system with a parametrically excited pendulum, Journal of Sound and Vibration 259 (2003), no. 4, 747-759.

[8] A. Tondl, T. Ruijgrok, F. Verhulst, and R. Nabergoj, Autoparametric Resonance in Mechanical Systems, Cambridge University Press, Cambridge, 2000.

[9] F. Verhulst, Autoparametric resonance, survey and new results, 2nd European Nonlinear Oscillation Conference (Prague, 1996), vol. 1, European Mechanics Society, 1996, pp. 483-488. 
[10] A. Vyas and A. K. Bajaj, Dynamics of autoparametric vibration absorbers using multiple pendulums, Journal of Sound and Vibration 246 (2001), no. 1, 115-135.

[11] J. Warminski, J. M. Balthazar, and R. M. L. R. F. Brasil, Vibrations of a non-ideal parametrically and self-excited model, Journal of Sound and Vibration 245 (2001), no. 2, 363-374.

J. Warminski: Department of Applied Mechanics, Lublin University of Technology, Nadbystrzycka 36, 20-618 Lublin, Poland

E-mail address: j.warminski@pollub.pl

K. Kecik: Department of Applied Mechanics, Lublin University of Technology, Nadbystrzycka 36, 20-618 Lublin, Poland

E-mail address: k.kecik@pollub.pl 


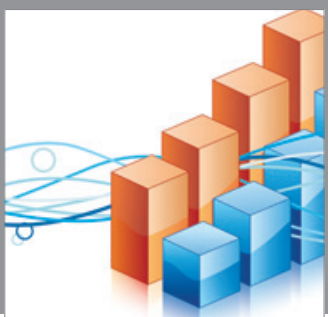

Advances in

Operations Research

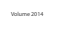

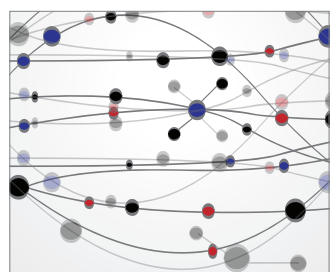

\section{The Scientific} World Journal
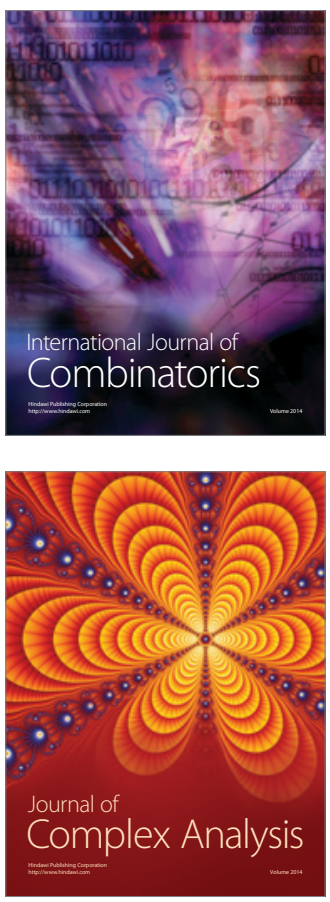

International Journal of

Mathematics and

Mathematical

Sciences
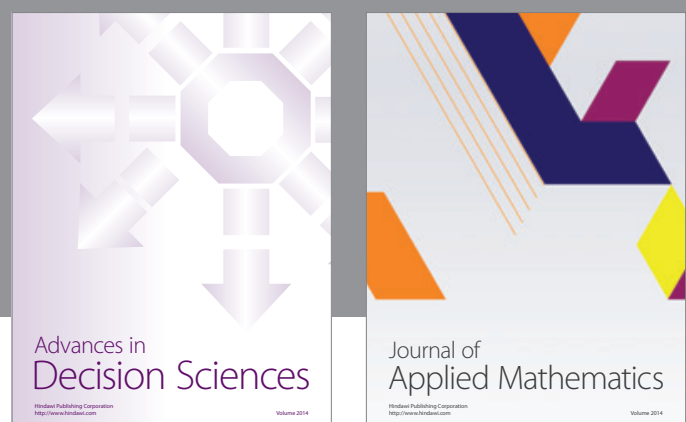

Journal of

Applied Mathematics
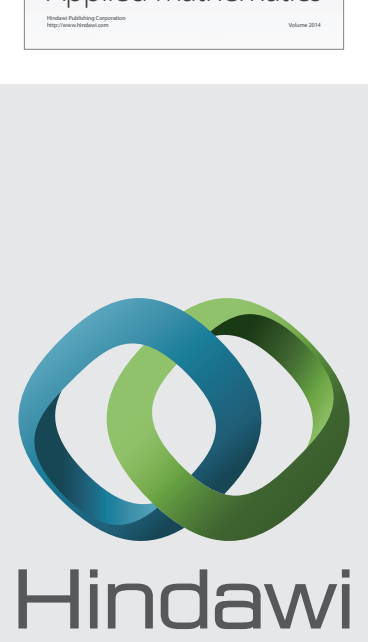

Submit your manuscripts at http://www.hindawi.com
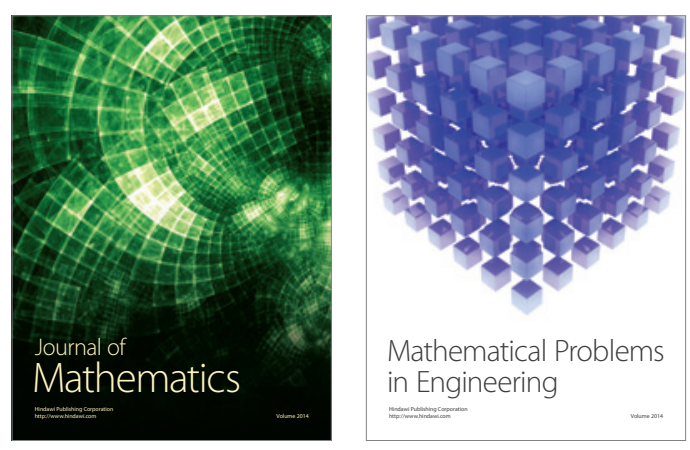

Mathematical Problems in Engineering
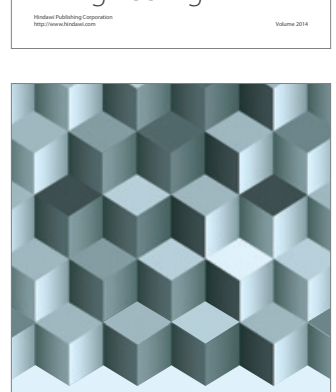

Journal of

Function Spaces
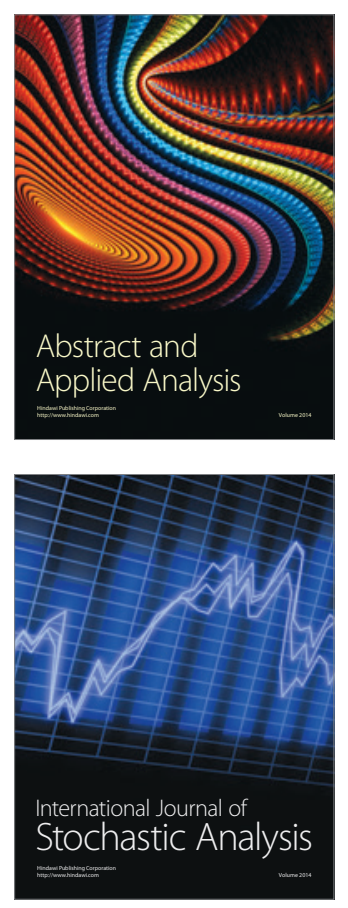

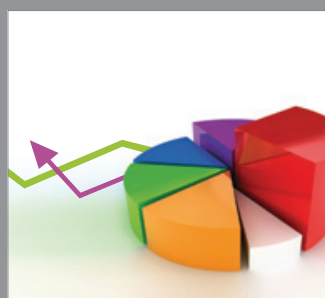

ournal of

Probability and Statistics

Promensencen
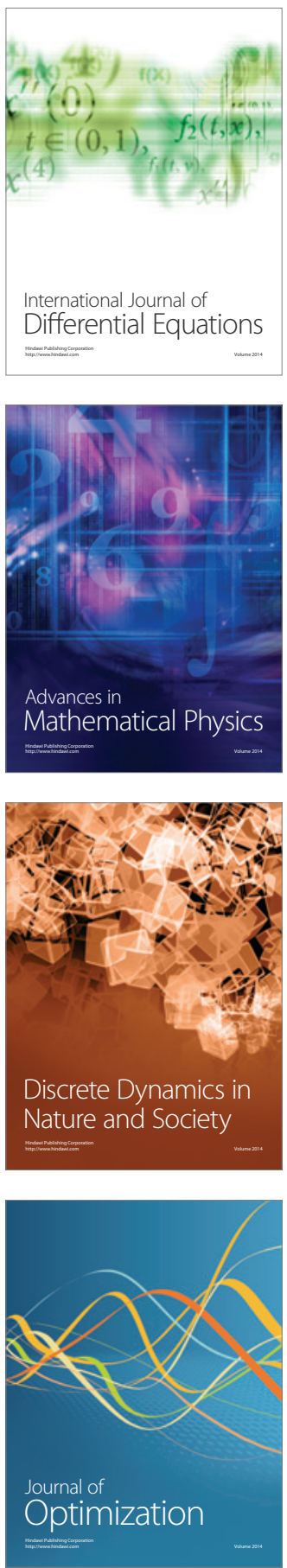